Editorial

\title{
Focus on Plasma Processes
}

The 17th International Colloquium on Plasma Processes (CIP09) was held in Marseille (France) from 22 to 26 June 2009. The CIP09 was organized by the French Vacuum Society (SFV), together with the International Exhibition on Vacuum and Surface Engineering (SVTM 2009) and the 37th A3TS Congress devoted to surface treatment.

The CIP is a biennial international conference that focuses on the latest developments in plasma processing science and technology. Although in its early years this Colloquium was essentially oriented towards the French plasma physics community, the CIP has evolved to become a well-established international conference in the area of plasma processes, covering issues from fundamentals to applications. To this end, a special attention has always been given not only to the understanding of plasma-surface interactions, including the relationship between the plasma parameters and the structural and functional properties of the plasma-treated films, but also to the development and modelling of new plasma sources. Moreover, in every edition, the CIP tries to feature new issues and challenges related with plasma processes in various industrial fields (microelectronics, micro-nanotechnology, aeronautics, mechanics, energy and environmental technology, life science), enhancing the opportunity for a fruitful scientific discussion and exchange of ideas. Presently, the CIP09 features the following list of ten topics:

- Advanced plasma sources,

- Plasma diagnostics and modelling,

- Multiphase plasmas,

- Plasma deposition processes,

- Plasma for functional and multifunctional coatings,

- Plasma for life science,

- Plasma for surface engineering,

- Plasma for microelectronics, micro and nanotechnology,

- Plasma for energy and environmental applications,

- Low temperature plasmas for ITER.

As associated editors of the European Physical Journal Applied Physics (Eur. Phys. J. Appl. Phys.), and guest editors for this special issue, we are pleased to publish a selected number of peer-reviewed papers based on different original contributions (in both oral and poster form) to the CIP09. These papers constitute a well-balanced representation of the topics treated during the conference, and their publication in the Eur. Phys. J. Appl. Phys. will significantly contribute to broaden the audience interested in the contents of the CIP.

We would like to thank all the authors for their co-operation and efforts in preparing and submitting, within suitable page length and deadline, the papers for this special issue of the Eur. Phys. J. Appl. Phys. devoted to the CIP09. 